\title{
Effect of Friction Reynolds Number on Turbulence Modulations in Liquid Flow in the Presence of Dust Particles in a Rotating System
}

\author{
Asaduzzaman ${ }^{1}$ and Dr.Md.Lutfor Rahman ${ }^{2}$ \\ Lecturer $^{1}$ and Professor ${ }^{2}$ \\ ${ }^{1}$ Department of Mathematics, Faculty of Science \& Engineering \\ North Bengal International University \\ Rajshahi, Rajshahi \\ Bangladesh
}

\begin{abstract}
The equation of liquid flow motion for dusty fluid turbulent flow in a rotating system has been derived by taking an average procedure, which includes the effect of dust particles, Coriolis force due to rotation and pressure velocity fluctuations at two points of the fluid flow. The rotating system includes the effect of friction Reynolds number on Coriolis force due to the rotation in the fluid flow with the correlation between pressure fluctuations and velocity fluctuations at two points of the flow field. It is clear that turbulence modulations have an effect on the impulse service of Reynolds number and dust particle in a rotating system.
\end{abstract}

Keywords: Dust particle, Turbulent flow, Corioles force, Centrifugal force, Tensor Correlations, rotating system and Friction Reynolds number.

\section{INTRODUCTION}

The fundamental characteristic of turbulence is that turbulent fluctuations are random in nature. In geophysical flows, the system is usually rotating with a constant angular velocity. Effect of Reynolds number on turbulence modulations in liquid flow with presence of dust particles in a rotating system can be found in many areas of industry, such as the production of the composite materials, chemical supplying pipes, environmental engineering, drinking and joust supplying industry, pharmaceuticals industry and Gas-solid suspensions industry and so on. Effect of Reynolds number on turbulence modulations in a liquid flow subject to rotation about the spanwise direction displays several phenomena of interest to engineering applications and turbulence modeling. Effect of rotation on flow and turbulence are in this case non-trivial. Liquid flow of pipes in any industry is unaffected by rotation if fluid motions are two dimensional and perpendicular to the rotation axes. Experiments of fully developed turbulent plane channel flow subject to spanwise rotation by Johnston et al. (1972) at $R_{e}=U_{b} h / \gamma=5750, R_{0}=2 \Omega h / U_{b} \leq 0.21$ and $R_{e}=$ $17500, R_{0} \leq 0.081$, and by Nakabayashi \& Kite(2005) at $R_{e} \leq 2750$ and $R_{0} \leq 0.056$, Where $U_{b}$ is the bulk mean velocity, h channel half-width, $\gamma$ viscosity and $\Omega$ rotation rate, have shown that turbulence is suppressed on one side of the channel where $\mathrm{S}>0$ and augmented on the other side where $-1<S<0$, in accordance with the discussion above. These sides are from now on called the stable and unstable side, respectively.DNS of spanwise rotating channel flow by Kristoffersen \& Andersson (1993) at $R e_{\tau}=194$ and $R o_{\tau} \leq 0.5$, Liu and Lu (2006) at $R e_{\tau}=194$ and $R o_{\tau} \leq 7.5$, Where $R e_{\tau}$ and $R o_{\tau}$ are based on the friction velocity instead of $U_{b}$, Where consistent with the experimental observations. At sufficiently high Reynolds number of the suppression of turbulence is so strong that the flow becomes laminar-like on the stable side. Particle-Turbulence interaction is central to many processes, both 
occurring in nature and in industrial applications such as sediment and nutrient transport in rivers and oceans, pollutant dispersion in the atmosphere, fuel mixing in combustion engines, flow of fiber suspension in paper making, etc. Recent studies on particle-flow interaction have led to significant advances in predicting small particles in highReynolds number turbulence. When the motion is referred to axes, which rotate steadily with the bulk of the fluid, the coriolis and centrifugal force must be supposed to act on the fluid. The Coriolis force due to rotation plays an important role in a rotating system of turbulent flow. The behavior of fuel mixing in combustion engines with presence of dust particles in a turbulent fluid depends on the concentration of the particles and on the size of the particles with respect to the scale of turbulent fluid. Besides one-point statistics, they studied the effect of rotation on turbulence modulations structures and Reynolds stress budgets. They observed that at high Reynolds number turbulence was weak as well on the unstable side, suggesting that the flow fully relaminarizes at sufficiently high Reynolds number. The critical rotation number $R o_{c}$ is a monotonic function of $R_{e}$,i.e. $R o_{c}=2.80$ for $R_{e}=$ 10000 and $R o_{c} \rightarrow 3.0$ for $R_{e} \rightarrow \propto$. The previous studies of turbulence modulations rotating system in a liquid flow were mostly limited to low Reynolds numbers, $R e_{\tau} \leq 194$, meaning that the influence of the Reynolds number on the statistics, turbulent structures and relaminarization on the stable side can be significant.

\section{MATHEMATICAL MODEL OF THE PROBLEM}

The Navies-Stokes equation of motion and continuity for viscous incompressible dusty fluid DNS velocity field of the liquid calculated is given by

$\frac{\partial u_{i}}{\partial x_{i}}=0$

$\frac{\partial u_{i}}{\partial t}+u_{j} \frac{\partial u_{i}}{\partial x_{j}}=-\frac{\partial \rho}{\partial x_{i}}+\delta_{1 i}+\frac{1}{R e_{\tau}} \nabla^{2} u_{i}-\bar{f}_{l}$

In presence of dust particles the equations of motion are given by

$\frac{\partial u_{i}}{\partial t}+u_{j} \frac{\partial u_{i}}{\partial x_{j}}=-\frac{\partial \rho}{\partial x_{i}}+\delta_{1 i}+\frac{1}{R e_{\tau}} \nabla^{2} u_{i}-\bar{f}_{l}+\frac{K N}{\rho}\left(\gamma_{i}-u_{i}\right)$

$\frac{\partial u_{i}}{\partial t}+u_{j} \frac{\partial u_{i}}{\partial x_{j}}=-\frac{\partial \rho}{\partial x_{i}}+\delta_{1 i}+\frac{1}{R e_{\tau}} \nabla^{2} u_{i}-\bar{f}_{l}+f_{c}\left(\gamma_{i}-u_{i}\right)$

In rotating systems, the equation of motion (4) becomes,

$\frac{\partial u_{i}}{\partial t}+u_{j} \frac{\partial u_{i}}{\partial x_{j}}=-\frac{\partial \rho}{\partial x_{i}}+\delta_{1 i}+\frac{1}{R e_{\tau}} \nabla^{2} u_{i}-\bar{f}_{l}+f_{c}\left(\gamma_{i}-u_{i}\right)-2\left(\Omega_{\mathrm{i}} \mathrm{u}_{\mathrm{i}} \eta_{\mathrm{i}}\right) \sin \theta$

$\frac{\partial u_{i}}{\partial t}+u_{j} \frac{\partial u_{i}}{\partial x_{j}}=-\frac{\partial \rho}{\partial x_{i}}+\delta_{1 i}+\xi \nabla^{2} u_{i}-\bar{f}_{l}+f_{c}\left(\gamma_{i}-u_{i}\right)-2\left(\Omega_{\mathrm{i}} \mathrm{u}_{\mathrm{i}} \eta_{\mathrm{i}}\right) \sin \theta$

Where, $\xi=\frac{1}{R e_{\tau}}$, friction Reynolds number on tensor .

$f_{c}=\frac{K N}{\rho}$, dimension of frequency.

$\mathrm{K}=$ the stokes's resistance coefficient which for spherical particle of radius $\mathrm{r}$ is $6 \pi \mu r$.

$\mathrm{N}=$ constant number of density of a dust particle.

$\rho=$ The density of the material in the dust particle.

$\delta_{1 i}=$ Average pressure gradient.

$\bar{f}_{l}=$ Local average interfacial force.

$\gamma_{i}=$ Solid particle of dust. 
$u_{i}=$ Fluid particle of dust.

$-2\left(\Omega_{i} u_{i} \eta_{i}\right) \sin \theta=-2(\bar{\Omega} \times \bar{u})$ Is the coriolis force in which $\Omega$ is the angular velocity?

$\mathrm{u}=$ the kinematical viscosity of the suspending fluid.

$\eta=$ is the unit vector perpendicular to $\bar{\Omega}$ and $\bar{u}$.

$\theta=$ is the angle between $\bar{\Omega}$ and $\bar{u}$.

We assume that the mean velocity $\bar{U}_{l}$ is constant throughout the region considered and independent of time and we put

$$
\left(U_{i}=\bar{U}_{l}+u_{i}\right)_{A}
$$

And

$$
\left(U_{j}=\bar{U}_{J}+u_{j}\right)_{B}
$$

The value of each term can be taken by using the equations of motion for $u_{j}$ at the point $\mathrm{B}$ and for $u_{i}$ at the point $\mathrm{A}$.

The equation of motion for $u_{i}$ at the point $\mathrm{A}$, obtained by the equation (6) is given by

$\frac{\partial u_{i}}{\partial t}+\left(\overline{U_{k}}+u_{k}\right) \frac{\partial u_{i}}{\partial x_{k}}=-\frac{\partial \rho}{\partial x_{i}}+\delta_{1 i}+\xi \nabla^{2} u_{i}-\bar{f}_{l}+f_{c}\left(\gamma_{i}-u_{i}\right)-2\left(\Omega_{\mathrm{i}} \mathrm{u}_{\mathrm{i}} \eta_{\mathrm{i}}\right) \sin \theta$

For an incompressible flow, $\left(u_{i} \frac{\partial u_{k}}{\partial x_{k}}\right)_{A}=0$

So that we can add this term in equation (7) and the equation becomes,

$\frac{\partial}{\partial t}\left(u_{i}\right)_{A}+\left[\overline{U_{k}}+\left(u_{k}\right)_{A}\right]\left(\frac{\partial}{\partial x_{k}}\right)_{A}\left(u_{i}\right)_{A}+\left(u_{i} \frac{\partial u_{k}}{\partial x_{k}}\right)_{A}=-\left(\frac{\partial}{\partial x_{i}}\right)_{A} \rho_{A}+\left(\delta_{1 \mathrm{i}}\right)_{\mathrm{A}}+\xi \nabla^{2}\left(u_{i}\right)_{A}-\overline{\left(f_{l}\right)_{A}}+f_{c}\left(\gamma_{i}-u_{i}\right)_{A}-$ $2\left(\Omega_{\mathrm{i}} \mathrm{u}_{\mathrm{i}} \eta_{\mathrm{i}}\right)_{A} \sin \theta$

Multiplying both sides by $\left(\mathrm{u}_{\mathrm{j}}\right)_{\mathrm{B}}$, from equation (8) is given by

$$
\begin{aligned}
& \left(u_{j}\right)_{B} \frac{\partial}{\partial t}\left(u_{i}\right)_{A}+\left[\overline{U_{k}}+\left(u_{k}\right)_{A}\right]\left(\frac{\partial}{\partial x_{k}}\right)_{A}\left(u_{i}\right)_{A}\left(u_{j}\right)_{B}+\left(\mathrm{u}_{\mathrm{i}}\right)_{\mathrm{A}}\left(\frac{\partial}{\partial x_{k}}\right)_{A}\left(u_{k}\right)_{A}\left(u_{j}\right)_{B}=-\left(\frac{\partial}{\partial x_{i}}\right)_{A} \rho_{A}\left(u_{j}\right)_{B}+\left(\delta_{1 \mathrm{i}}\right)_{\mathrm{A}}\left(u_{\mathrm{j}}\right)_{B}+ \\
& \xi \nabla^{2}\left(u_{i}\right)_{A}\left(\mathrm{u}_{\mathrm{j}}\right)_{\mathrm{B}}-\overline{\left(f_{l}\right)_{A}}\left(\mathrm{u}_{\mathrm{j}}\right)_{\mathrm{B}}+f_{C}\left(\gamma_{i}-u_{i}\right)_{A}\left(\mathrm{u}_{\mathrm{j}}\right)_{\mathrm{B}}-2\left(\Omega_{\mathrm{i}} \mathrm{u}_{\mathrm{i}} \eta_{\mathrm{i}}\right)_{A} \sin \theta\left(\mathrm{u}_{\mathrm{j}}\right)_{\mathrm{B}}
\end{aligned}
$$

Where, $\left(\mathrm{u}_{\mathrm{j}}\right)_{\mathrm{B}}$ can be treated as a constant in a differential process at the point $\mathrm{A}$.

Again, the equation of motion for $u_{j}$ at the point $\mathrm{B}$, obtained by the equation (6) is given by

$\frac{\partial u_{j}}{\partial t}+\left(\overline{U_{k}}+u_{k}\right) \frac{\partial u_{j}}{\partial x_{k}}=-\frac{\partial \rho}{\partial x_{j}}+\delta_{1 j}+\xi \nabla^{2} u_{j}-\bar{f}_{J}+f_{c}\left(\gamma_{j}-u_{j}\right)-2\left(\Omega_{\mathrm{j}} \mathrm{u}_{\mathrm{j}} \eta_{\mathrm{j}}\right) \sin \theta$

For an incompressible flow, $\left(u_{j} \frac{\partial u_{k}}{\partial x_{k}}\right)_{B}=0$

So that we can add this term in equation (10) and the equation becomes,

$\frac{\partial}{\partial t}\left(u_{j}\right)_{B}+\left[\overline{U_{k}}+\left(u_{k}\right)_{B}\right]\left(\frac{\partial}{\partial x_{k}}\right)_{B}\left(u_{j}\right)_{B}+\left(u_{j} \frac{\partial u_{k}}{\partial x_{k}}\right)_{B}=-\left(\frac{\partial}{\partial x_{j}}\right)_{B} \rho_{B}+\left(\delta_{1 j}\right)_{B}+\xi \nabla^{2}\left(u_{j}\right)_{B}-\overline{\left(f_{j}\right)_{B}}+f_{c}\left(\gamma_{j}-u_{j}\right)_{B}-$ $2\left(\Omega_{\mathrm{j}} \mathrm{u}_{\mathrm{j}} \eta_{\mathrm{j}}\right)_{B} \sin \theta$

Multiplying both sides by $\left(u_{i}\right)_{A}$, from equation (11) is given by 


$$
\begin{aligned}
& \left(u_{i}\right)_{A} \frac{\partial}{\partial t}\left(u_{j}\right)_{B}+\left[\overline{U_{k}}+\left(u_{k}\right)_{B}\right]\left(\frac{\partial}{\partial x_{k}}\right)_{B}\left(u_{j}\right)_{B}\left(u_{i}\right)_{A}+\left(u_{j}\right)_{B}\left(\frac{\partial}{\partial x_{k}}\right)_{B}\left(u_{k}\right)_{B}\left(u_{i}\right)_{A}=-\left(\frac{\partial}{\partial x_{j}}\right)_{B} \rho_{B}\left(u_{i}\right)_{A}+\left(\delta_{1 j}\right)_{B}\left(u_{i}\right)_{A}+ \\
& \xi \nabla^{2}\left(u_{j}\right)_{B}\left(u_{i}\right)_{A}-\overline{\left(f_{J}\right)_{B}}\left(u_{i}\right)_{A}+f_{c}\left(\gamma_{j}-u_{j}\right)_{B}\left(u_{i}\right)_{A}-2\left(\Omega_{\mathrm{j}} u_{\mathrm{j}} \eta_{\mathrm{j}}\right)_{B} \sin \theta\left(u_{i}\right)_{A}
\end{aligned}
$$

Where, $\left(u_{i}\right)_{A}$ can be treated as a constant in a differential process at the point $\mathrm{B}$.

Now, adding the equations (9) and (12) and we can get the result

$\frac{\partial}{\partial t}\left[\left(u_{i}\right)_{A}\left(u_{j}\right)_{B}\right]+\left[\left(\frac{\partial}{\partial x_{k}}\right)_{A}\left(u_{i}\right)_{A}\left(u_{k}\right)_{A}\left(u_{j}\right)_{B}+\left(\frac{\partial}{\partial x_{k}}\right)_{B}\left(u_{j}\right)_{B}\left(u_{k}\right)_{B}\left(u_{i}\right)_{A}\right]+$

$\overline{U_{k}}\left[\left(\frac{\partial}{\partial x_{k}}\right)_{A}\left(u_{i}\right)_{A}\left(u_{j}\right)_{B}+\left(\frac{\partial}{\partial x_{k}}\right)_{B}\left(u_{j}\right)_{B}\left(u_{i}\right)_{A}\right]=$

$-\left[\left(\frac{\partial}{\partial x_{i}}\right)_{A} \rho_{A}\left(u_{j}\right)_{B}+\left(\frac{\partial}{\partial x_{j}}\right)_{B} \rho_{B}\left(u_{i}\right)_{A}\right]+\left[\left(\delta_{1 \mathrm{i}}\right)_{\mathrm{A}}\left(u_{j}\right)_{B}+\left(\delta_{1 j}\right)_{B}\left(u_{i}\right)_{A}\right]+\xi\left[\nabla^{2}\left(u_{i}\right)_{A}\left(\mathrm{u}_{\mathrm{j}}\right)_{\mathrm{B}}+\nabla^{2}\left(u_{j}\right)_{B}\left(u_{i}\right)_{A}\right]-$

$\left[\overline{\left(f_{l}\right)_{A}}\left(\mathrm{u}_{\mathrm{j}}\right)_{\mathrm{B}}+\overline{\left(f_{j}\right)_{B}}\left(u_{i}\right)_{A}\right]+f_{C}\left[\left(\gamma_{i}-u_{i}\right)_{A}\left(\mathrm{u}_{\mathrm{j}}\right)_{\mathrm{B}}+\left(\gamma_{j}-u_{j}\right)_{B}\left(u_{i}\right)_{A}\right]-2\left[\left(\Omega_{\mathrm{i}} \mathrm{u}_{\mathrm{i}} \eta_{\mathrm{i}}\right)_{A}\left(\mathrm{u}_{\mathrm{j}}\right)_{\mathrm{B}}+\left(\Omega_{\mathrm{j}} \mathrm{u}_{\mathrm{j}} \eta_{\mathrm{j}}\right)_{B}\left(u_{i}\right)_{A}\right] \sin \theta$

We can find the relation of turbulence modulations in a liquid flow with presence of dust particles in a rotating system at the point $\mathrm{B}$ to those at point $\mathrm{A}$, it will give no difference if we take one point as the origin of $\mathrm{A}$ or $\mathrm{B}$ of the coordinate system.

Let us consider the point $\mathrm{A}$ as the origin and we can write

$$
\varsigma_{k}=\left(x_{k}\right)_{B}-\left(x_{k}\right)_{A}
$$

Then we obtain $\left(\frac{\partial}{\partial x_{k}}\right)_{A}=-\frac{\partial}{\partial \zeta_{k}},\left(\frac{\partial}{\partial x_{k}}\right)_{B}=\frac{\partial}{\partial \zeta_{k}}$

$$
\left(\frac{\partial^{2}}{\partial x_{k} \partial x_{k}}\right)_{A}=\left(\frac{\partial^{2}}{\partial x_{k} \partial x_{k}}\right)_{B}=\frac{\partial^{2}}{\partial \zeta_{k} \partial \zeta_{k}}
$$

Using the above relations in equation (13) and taking ensemble average on both sides, then the equation (13) becomes

$$
\begin{aligned}
& \frac{\partial}{\partial t} \overline{\left(u_{\imath}\right)_{A}\left(u_{\jmath}\right)_{B}}-\frac{\partial}{\partial \varsigma_{k}} \overline{\left(u_{\imath}\right)_{A}\left(u_{k}\right)_{A}\left(u_{\jmath}\right)_{B}}+\frac{\partial}{\partial \varsigma_{k}} \overline{\left(u_{\imath}\right)_{A}\left(u_{k}\right)_{B}\left(u_{\jmath}\right)_{B}}=-\left[-\frac{\partial}{\partial \varsigma_{k}} \overline{\rho_{A}\left(u_{\jmath}\right)_{B}}+\frac{\partial}{\partial \varsigma_{k}} \overline{\rho_{B}\left(u_{l}\right)_{A}}\right]+ \\
& {\left[\overline{\left(\delta_{1 \imath}\right)_{A}\left(u_{\jmath}\right)_{B}}+\overline{\left(\delta_{1 \jmath}\right)_{B}\left(u_{\imath}\right)_{A}}\right]+2 \xi \nabla^{2}\left[\overline{\left(u_{\imath}\right)_{A}\left(u_{\jmath}\right)_{B}}\right]+\left[\overline{\overline{\left(\bar{f}_{\imath}\right)_{A}}\left(u_{\jmath}\right)_{B}}+\overline{\left(\overline{f_{J}}\right)_{B}\left(u_{\imath}\right)_{A}}\right]+f_{c}\left[\overline{\left(\gamma_{l}\right)_{A}\left(u_{\jmath}\right)_{B}}-2 \overline{\left(u_{\imath}\right)_{A}\left(u_{\jmath}\right)_{B}}+\right.} \\
& \left.\overline{\left(u_{\imath}\right)_{A}\left(\gamma_{J}\right)_{B}}\right]-2\left[\overline{\left(\Omega_{1} \mathrm{u}_{1} \eta_{1}\right)_{A}\left(u_{\mathrm{J}}\right)_{B}}+\overline{\left(\Omega_{\mathrm{J}} \mathrm{u}_{\mathrm{j}} \eta_{\mathrm{j}}\right)_{B}\left(u_{l}\right)_{A}}\right] \sin \theta
\end{aligned}
$$

Equation (14) represents the equation of turbulence modulations in a liquid flow in rotating system under the influence of dust particles with pressure-velocity correlation.

It is noted that the coefficient of $\overline{U_{k}}$ has been chancellors'. The equation (14) details the turbulence modulations in liquid flow in a rotating system with presence of dust particles, where the motions with respect to a coordinate system moving with $\overline{U_{k}}$. Equation (14) contains the double velocity correlations $\overline{\left(u_{l}\right)_{A}\left(u_{J}\right)_{B}}$, double velocity correlation between dust particles and fluid motion such as $\overline{\left(\gamma_{l}\right)_{A}\left(u_{\jmath}\right)_{B}}$ and $\overline{\rho_{A}\left(u_{\jmath}\right)_{B}}$ triple correlations such as $\overline{\left(u_{\imath}\right)_{A}\left(u_{k}\right)_{A}\left(u_{J}\right)_{B}}$ where all the terms apart from one to another. The correlations $\overline{\rho_{A}\left(u_{\jmath}\right)_{B}}$ and $\overline{\rho_{B}\left(u_{l}\right)_{A}}$ form the tensors of the first order, because pressure is a scalar quantity and the triple correlations $\overline{\left(u_{\imath}\right)_{A}\left(u_{k}\right)_{A}\left(u_{J}\right)_{B}}$ and $\overline{\left(u_{\imath}\right)_{A}\left(u_{k}\right)_{B}\left(u_{J}\right)_{B}}$ are third order tensors.

We shall give a name to the first correlations by $\left(R_{p, j}\right)_{A, B}$ and second order correlations by $\left(T_{i, j}\right)_{A, B}$ and third order correlations by $\left(V_{i k, j}\right)_{A, B}$. Hence the set, 


$$
\begin{aligned}
& \left(R_{i, p}\right)_{A, B}=\overline{\left(u_{\imath}\right)_{A} \rho_{B}}, \quad\left(R_{p, j}\right)_{A, B}=\overline{\rho_{A}\left(u_{\mathrm{j}}\right)_{B}}, \quad\left(V_{i k, j}\right)_{A, B}=\overline{\left(\mathrm{u}_{\mathrm{i}}\right)_{\mathrm{A}}\left(\mathrm{u}_{\mathrm{k}}\right)_{\mathrm{A}}\left(\mathrm{u}_{\mathrm{\jmath}}\right)_{\mathrm{B}}}, \quad \quad\left(V_{i, k j}\right)_{A, B}= \\
& \overline{\left(u_{\imath}\right)_{A}\left(u_{k}\right)_{B}\left(u_{\jmath}\right)_{B}}, \quad\left(T_{i, j}\right)_{A, B}=\overline{\left(u_{\imath}\right)_{A}\left(u_{\jmath}\right)_{B}}, \quad\left(O_{i, j}\right)_{A, B}=\overline{\left(\gamma_{l}\right)_{A}\left(u_{\jmath}\right)_{B}}, \\
& \left(S_{i, j}\right)_{A, B}=\overline{\left(u_{l}\right)_{A}\left(\gamma_{\jmath}\right)_{B}}, \quad\left(C_{i, j}\right)_{A, B}=\overline{\left(\delta_{1 l}\right)_{A}\left(u_{\jmath}\right)_{B}}, \quad\left(C_{j, i}\right)_{A, B}=\overline{\left(\delta_{1 \jmath}\right)_{B}\left(u_{\imath}\right)_{A}}, \\
& \left(F_{i, j}\right)_{A, B}=\overline{\overline{\left(f_{l}\right)_{A}}\left(u_{J}\right)_{B}} \quad,\left(F_{j, i}\right)_{A, B}=\overline{\left(\overline{f_{J}}\right)_{B}\left(u_{\imath}\right)_{A}}
\end{aligned}
$$

Where the exponent $\mathrm{P}$ strikes the pressure and is not a shape exponent like $\mathrm{i}$ or $\mathrm{j}$. so that the concurrence symposium does not apply to $\mathrm{p}$. also the term $\overline{\left(\Omega_{1} \mathrm{u}_{1} \eta_{1}\right)_{A}\left(u_{\mathrm{J}}\right)_{B}}$ and $\overline{\left(\Omega_{\mathrm{j}} \mathrm{u}_{\mathrm{j}} \eta_{\mathrm{j}}\right)_{B}\left(u_{\imath}\right)_{A}}$ from the tensor of second order and we shall give a name to these by $E_{i, j}$ and $L_{i, j}$ respectively. Thus we get

$$
\left(E_{i, j}\right)_{A, B}=\overline{\left(\Omega_{1} \mathrm{u}_{1} \eta_{\mathrm{1}}\right)_{A}\left(u_{\mathrm{J}}\right)_{B}},\left(L_{i, j}\right)_{A, B}=\overline{\left(\Omega_{\mathrm{j}} \mathrm{u}_{\mathrm{j}} \eta_{\mathrm{j}}\right)_{B}\left(u_{\imath}\right)_{A}}
$$

If we use the above relations of first, second and third order correlations in equation (14). Then we get,

$\frac{\partial}{\partial t}\left(T_{i, j}\right)_{A, B}-\frac{\partial}{\partial \xi_{k}}\left(V_{i k, j}\right)_{A, B}+\frac{\partial}{\partial \xi_{k}}\left(V_{i, k j}\right)_{A, B}=-\left[-\frac{\partial}{\partial \xi_{k}}\left(R_{p, j}\right)_{A, B}+\frac{\partial}{\partial \xi_{k}}\left(R_{i, p}\right)_{A, B}\right]+\left[\left(C_{i, j}\right)_{A, B}+\left(C_{j, i}\right)_{A, B}\right]+$

$2 \gamma \nabla^{2}\left[\left(T_{i, j}\right)_{A, B}\right]+\left[\left(F_{i, j}\right)_{A, B}+\left(F_{j, i}\right)_{A, B}\right]+f_{c}\left[\left(O_{i, j}\right)_{A, B}-2\left(T_{i, j}\right)_{A, B}+\left(S_{i, j}\right)_{A, B}\right]-2\left[\left(E_{i, j}\right)_{A, B}+\left(L_{i, j}\right)_{A, B}\right] \sin \theta$

Where all correlations refer to the two points A and B. Now for an isotropic turbulence modulations of an incompressible flow, the double pressure-velocity correlations are zero.

i.e.

$$
\left(R_{p, j}\right)_{A, B}=\left(R_{i, p}\right)_{A, B}=0 .
$$

In isotropic turbulence modulations it follows from the condition of invariance under reflection with respect to point A.

$$
\begin{gathered}
\overline{\left(u_{\imath}\right)_{A}\left(u_{k}\right)_{B}\left(u_{\jmath}\right)_{B}}=-\overline{\left(\mathrm{u}_{\mathrm{l}}\right)_{\mathrm{A}}\left(\mathrm{u}_{\mathrm{k}}\right)_{\mathrm{A}}\left(\mathrm{u}_{\mathrm{\jmath}}\right)_{\mathrm{B}}} \\
\left(V_{i, k j}\right)_{A, B}=-\left(V_{i k, j}\right)_{A, B}
\end{gathered}
$$

Thus the equation (14) becomes

$\frac{\partial}{\partial t}\left(T_{i, j}\right)_{A, B}-\frac{\partial}{\partial \xi_{k}}\left[\left(V_{i k, j}\right)_{A, B}+\left(V_{i, k j}\right)_{A, B}\right]=\left[\left(C_{i, j}\right)_{A, B}+\left(C_{j, i}\right)_{A, B}\right]+2 \gamma \nabla^{2}\left[\left(T_{i, j}\right)_{A, B}\right]+\left[\left(F_{i, j}\right)_{A, B}+\left(F_{j, i}\right)_{A, B}\right]+$ $f_{c}\left[\left(O_{i, j}\right)_{A, B}-2\left(T_{i, j}\right)_{A, B}+\left(S_{i, j}\right)_{A, B}\right]-2\left[\left(E_{i, j}\right)_{A, B}+\left(L_{i, j}\right)_{A, B}\right] \sin \theta(16)$

The term $\left[\left(\frac{\partial}{\partial \xi_{\mathrm{k}}} \mathrm{V}_{\mathrm{ik}, \mathrm{j}}\right)_{\mathrm{A}, \mathrm{B}}+\left(\mathrm{V}_{\mathrm{i}, \mathrm{kj}}\right)_{\mathrm{A}, \mathrm{B}}\right],\left[\left(C_{i, j}\right)_{A, B}+\left(C_{j, i}\right)_{A, B}\right],\left[\left(F_{i, j}\right)_{A, B}+\left(F_{j, i}\right)_{A, B}\right]$ and $\left[\left(E_{i, j}\right)_{A, B}+\left(L_{i, j}\right)_{A, B}\right]$ from the second order tensors and we shall give a name to these by

$$
\begin{array}{ll}
V_{i, j}=\frac{\partial}{\partial \xi_{k}}\left[\left(V_{i k, j}\right)_{A, B}+\left(V_{i, k j}\right)_{A, B}\right], & C_{i, j}=\left[\left(C_{i, j}\right)_{A, B}+\left(C_{j, i}\right)_{A, B}\right] \\
F_{i, j}=\left[\left(F_{i, j}\right)_{A, B}+\left(F_{j, i}\right)_{A, B}\right] \quad, & W_{i, j}=\left[\left(E_{i, j}\right)_{A, B}+\left(L_{i, j}\right)_{A, B}\right]
\end{array}
$$

Hence the equation (16) becomes $\frac{\partial}{\partial t}\left(T_{i, j}\right)_{A, B}-V_{i, j}=C_{i, j}+2 \gamma \nabla^{2}\left[\left(T_{i, j}\right)_{A, B}\right]+F_{i, j}+f_{c}\left[\left(O_{i, j}\right)_{A, B}-2\left(T_{i, j}\right)_{A, B}+\left(S_{i, j}\right)_{A, B}\right]-2 W_{i, j} \sin \theta$ 
Equation (17) represents the equation of turbulence modulations of liquid flow in a rotating system under the influence of dust particles in terms of the second order tensor correlation.

In the absence of dust particles, then $f_{c}=0$ and equations (17) becomes

$\frac{\partial}{\partial t}\left(T_{i, j}\right)_{A, B}-V_{i, j}=C_{i, j}+2 \gamma \nabla^{2}\left[\left(T_{i, j}\right)_{A, B}\right]+F_{i, j}-2 W_{i, j} \sin \theta$

Equation (18) represents the turbulence modulations in a liquid flow in rotating system with the second order tensors correlation.

For non-rotating system, $W_{i, j}=0$ and equation (18) becomes

$\frac{\partial}{\partial t}\left(T_{i, j}\right)_{A, B}-V_{i, j}=C_{i, j}+2 \gamma \nabla^{2}\left[\left(T_{i, j}\right)_{A, B}\right]+F_{i, j}$

Hence the equation (19) represents the turbulence modulations in a liquid flow in non-rotating system with the second order tensors correlation.

\section{RESULT AND DISCUSSION}

Here we compare the results for two points of the fluid flow for two particles encumbered cases with the same volume and mass fraction but with different particle shapes to the single phase case. The aim here is to measure the effect of particle shape only on turbulence modulations. The equation of liquid flow motion for dusty fluid turbulent flow in a rotating system has been derived by taking average procedure, which includes the effect of dust particles, Coriolis force due to rotation and pressure velocity fluctuations at two points of the fluid flow. In order to study the effect of rotation on turbulence modulations structures in a quantitative way Kristoffersen \& Andersson (1993), Alvelius (1999) and Grundestam et al.(2008) computed two-point correlations at $R e_{\tau}=180$ at 194 . Generally, relatively short streamwise two-point correlations on the unstable side and relatively long ones on the other laminar-like side were observed when the channel was rotating. Here, I present spectra since they reveal better the impact of rotation on the different flow scales, especially the large scales which are known to become important at higher $R_{e}$ in wall flows (smits et al.2011).

The flows is on the strongly turbulent unstable side of the channel since spectra are less instructive for the stable side when relaminarization. At higher rotation rates large-scales structures become progressively less energetic. The energetic scales are thus becoming shorter at higher Reynolds number and there is no clear indication of roll cells observed in the spectra.

Accordingly, no large structures like roll cells are observed invisulizations. Results of the particle rotation rates measurements are reported here. Al-through this data have to be considered preliminary results, due to their limited statistical significance, it has to be pointed out that to the authors knowledge, until now, such data were only available in DNS. For each measurement we determined the instantaneous rotation rate vector, $\Omega=\left(\Omega_{x}, \Omega_{y}, \Omega_{z}\right)$ as explained in the mathematical model. Given the isotropy of the flow, it is reasonable to assume that also the three components of the rotation vector are isotropic; therefore, to increase the accuracy of our statistical estimates we combine the measurements of the three components in a single array called $\Omega$. Another quantity of interest is the magnitude of the rotation vector $|\Omega|$.The results shows that mean is centered at zero in both cases, which expected, given that the mean flow in the container is nearly zero. Instead, the mean of $|\Omega|$ has to be obviously larger than zero and it seems that ellipsoids rotate faster in average than sphere. This result can be explained by the fact that the longer axis of the ellipsoidal particles provide act as a longer level arm for the fluid to act on the particles, thus the available torque is larger than for spheres. The occurrence of the turbulent flow will depend on the values of the non-dimensional number known as critical Reynolds number, which varies from 2000 to 2300 . The flow will be turbulent if the Reynolds number $\left(R_{e}\right)$ is greater than the critical Reynolds number $\left(R e_{\tau}\right)$. So that the turbulent flow occurs at high Reynolds number. If the Reynolds number increases from 1600 to 2500 then the flow changes to turbulent flow from laminar 
flow, the impulse service of Reynolds number on turbulence modulations in liquid flow changes in a range. It is clear that turbulence modulations have effect on the impulse service of Reynolds number and dust particles in a rotating system. Since, the fluctuation velocity is isotropic, the translation and rotation of Reynolds number of liquid flow are also isotropic in turbulence modulations. The distribution function of turbulence modulations in liquid flow $\theta$ changes in the range from $0^{\circ}$ to $90^{\circ}$. In a rotating system angular velocity plays also important role in the flow field. Since the fluctuation of flow velocity gradient is random and changes around zero, then the angular velocity of turbulence fluctuations around zero. For a turbulent pipe flow, the turbulent intensity of velocity gradient on flow direction is stranger than that on lateral direction. Hence, velocity gradient on the flow direction leads to the angular velocity of turbulence modulations in liquid on $\mathrm{x}$ and $\mathrm{y}$ direction, while velocity gradient on the lateral direction leads to that on $\mathrm{z}$ direction. Thus the angular velocity of turbulence modulations in liquid on $\mathrm{x}$ and $\mathrm{y}$ direction is wider than that on $\mathrm{z}$ direction. In presence of dust particles this concentration leads to be weaker and impulse service of turbulence modulations of liquid flow becomes more uniform Reynolds number increases. In a rotating system, Coriolis force and centrifugal force act on the fluid. For a non-rotating system, the velocity of turbulence modulations has the same fluctuation property as fluid velocity due to the strong following ability of turbulence modulations in liquid flow. Thus the resulting equation demonstrates that in presence of dust particles the fluctuation velocity gradient strengths with the increases of Reynolds number due to rotation of turbulence modulations on liquid flow. In the second case we could speculate that due to their rotation, elongated particles tend to sustain vortices of a size larger or equal to their major axis. This would require a spatial resolution much higher than what presented here, which instead has been optimized to measure global effects on turbulence modulations. Hence, the analysis of velocity gradients is left as future work.

\section{CONCLUSIONS}

We have reported an experimental technique with which we can simultaneously Measure rotational rates of arbitrarily shaped particles and fluid velocity by combining standard stereoscopic particle image velocimetry and index of refraction matching.

\section{REFERENCE}

[1] DAVIS, R.E. (1969): On the high Reynolds number flow over a wavy boundary.J.Fluid Mech.36, 337-346.

[2] M.A.AL-ZANAIDI AND W.H.HUI., Turbulent airflow over water waves a numerical study. J, Fluid Mech.(1984)Vol148,PP.225-246.

[3] M.L.Rahman and M.S.Alam Sarker,Thermal Decay Process of MHD Turbulent Flow in a Rotating System in Presence of Dust Particles,Rajshahi University Studies,Part-B,Journal of Sciences, Vol-33,65-78(2005).

[4] Norberg, C., Effects of Reynolds Number and a Low-Intensity Free Stream Turbulence on the Flow around a Circular Cylinder. Chalmers University of Technology, ISSN02809265, 1987.

[5] M.S.Alam Sarker., Thermal Decay Process oh MHD turbulence in a rotating system. Rajshahi University Studies, Part-B, Vol-25,139-152(1997).

\section{AUTHORS' CONTRIBUTIONS:}

This work was carried out in collaboration between both authors. The author Asaduzzaman gathered the initial data and helps to analysis those data, drafting the article and interprets the results. The author L.Rahman designed this study. This author created the conception how to write the diagram, Solution of mathematical problems, Motion of particle on rotating system and programming and interpreted the results. Both authors read and approved the final manuscript. 